\title{
El curso natural de los aneurismas cerebrales no rotos varía de acuerdo con su tamaño, ubicación y forma
}

The natural course of unruptured cerebral aneurysms varies according to its size, location and shape

Morita A y col. NEJM 2012;366:2474-82.

\section{Objetivo}

Dilucidar el curso natural de los aneurismas cerebrales no rotos (ACNR) e identificar los factores de riesgo específicos e independientes de ruptura.

\section{Diseño, lugar y pacientes}

Estudio prospectivo de cohortes, realizado en 283 centros de Japón. Se incluyeron (entre 2001 a 2004) 5.720 pacientes mayores de 20 años con diagnóstico reciente de ACNR $\geq 3 \mathrm{~mm}$. Fueron excluidos los pacientes que presentaran episodios previos de hemorragia intracraneal de causa desconocida o no tratada, puntuación de Rankin modificada mayor a 2, aneurismas fusiformes o disecantes y aneurismas de la porción cavemosa de la arteria carótida interna.

\section{Evaluación de factores pronósticos}

Se evaluaron el tamaño de los aneurismas, la ubicación y la presencia o ausencia de un saco hijo (protuberancia irregular de la pared del aneurisma) mediante angiorresonancia, angio-TC o angiografía digital convencional. Además se analizaron diferencias según sexo, hipertensión arterial, dislipidemia, tabaquismo, antecedentes familiares de hemorragia subaracnoidea (HSA), aneurismas múltiples, y trombosis o calcificación de los mismos.

\section{Resultados Principales.}

De los 6.697 aneurismas estudiados, el 91\% fueron hallazgos incidentales. Dos tercios de los pacientes fueron mujeres, con una media de edad de $62,5+/-10,3$ años. El tamaño medio de los aneurismas fue de $5,7 \pm 3,6 \mathrm{~mm}$, la mayoría ubicados en las arterias cerebrales medias $(36 \%)$ y arterias carótidas intemas $(34 \%)$. Durante un período de seguimiento que incluyó 11.660 aneurismaaños, las rupturas se documentaron en 111 aneurismas, con una tasa anual de 0,95\% (IC 95\% 0,79 a 1,15). Los principales resultados se resumen en la tabla 1. La presencia de otro aneunisma causando HSA, tabaquismo, antecedentes familiares de HSA, y la presencia de múltiples aneurismas no influyó en el riesgo de ruptura de un aneurisma $(P>0,20)$. Los aneurismas trombosados o calcificados tuvieron una mayor tendencia a la ruptura.
Tabla 1: Factores de riesgo asociados a la ruptura de aneunismas cerebrales

\begin{tabular}{|c|c|}
\hline Factor de Riesgo & Hazard Ratio (IC 95\%) \\
\hline Sexo femenino & $1,54(0,99$ a 2,42$)$ \\
\hline Edad mayor de 70 años & $1,21(0,81$ a 1,78$)$ \\
\hline Hipertensión arterial & $1,41(0,96$ a 2,07) \\
\hline Dislipidemia & $0,54(0,28$ a 1,03$)$ \\
\hline Aneurisma con saco hijo & $1,63(1,08$ a 2,48$)$ \\
\hline \multicolumn{2}{|l|}{ Tamaño de aneurisma } \\
\hline 3 a $4 \mathrm{~mm}$ & Referencia \\
\hline 5 a $6 \mathrm{~mm}$ & $1,13(0,8$ a 2,22) \\
\hline 7 a $9 \mathrm{~mm}$ & $3,35(1,87$ a 6,00$)$ \\
\hline 10 a $24 \mathrm{~mm}$ & $9,09(5,25$ a 15,74$)$ \\
\hline$>25 \mathrm{~mm}$ & $76,26$ (32,76 a 177,54$)$ \\
\hline \multicolumn{2}{|l|}{ Localización del aneurisma } \\
\hline Arteria cerebral media & Referencia \\
\hline Arteria comunicante anterior & $2,02(1,13$ a 3,58$)$ \\
\hline Arteria carótida interna & $0,43(0,18$ a 1,01$)$ \\
\hline Arteria comunicante posterior & $1,90(1,12$ a 3,21$)$ \\
\hline $\begin{array}{l}\text { Arteria cerebelosa superior, basilar y } \\
\text { punta basilar }\end{array}$ & $1,49(0,78$ a 2,83) \\
\hline $\begin{array}{l}\text { Arteria vertebral, cerebelosa } \\
\text { posteroinferior y unión vertebrobasilar }\end{array}$ & 0,68 (0,16 a 2,87) \\
\hline Otras & $1,48(0,61$ a 3,60$)$ \\
\hline
\end{tabular}

\section{Conclusión}

Este estudio mostró que el curso natural de los aneurismas cerebrales no rotos varía de acuerdo con el tamaño, la ubicación y la forma del aneunisma.

Fuente de financiamiento: Ministerio de Salud, Trabajo y Bienestar de Japón, entre otros.

\section{Comentario}

A pesar de las disparidades entre las poblaciones reportadas, los resultados obtenidos de este estudio no difieren significativamente de otras publicaciones, como la de Rinkel con una ruptura anual del 0,8\% (0,4 a 1\%), Juvela que informa 10,5\% (5,3 a 15,8\%) a los diez años o Werner con una ruptura anual 0,5 a 1,3\% ${ }^{1}$. Entre los factores que influyen en la tasa de ruptura, el tamaño del aneurisma es dominante ${ }^{1,2,3,8}$. La ubicación también afecta el riesgo de ruptura, siendo los de la circulación posterior los que presentan mayor riesgo ${ }^{2,8}$. La relación con la edad ${ }^{4,5,6}$ y la hipertensión arterial ${ }^{2,5,7,8}$ son controversiales. Algunos sostienen que factores de riesgo como tabaquismo ${ }^{8}$, antecedentes de hemorragia subaracnoidea y la multiplicidad de los aneurismas influyen en la factibilidad de la ruptura, sin encontrar diferencias significativas entre pacientes tratados y no tratados ${ }^{2}$. Tampoco hay consenso en relación al tratamiento.La Guía de la Organización Europea de Stroke sugiere que considerando el riesgo (peligro del procedimiento de 5 a $50 \%$, vs. riesgo de ruptura espontánea de 0 a 10\% por año) y el beneficio (la esperanza de vida, con o sin déficit menor), la decisión a favor o en contra de la intervención es una decisión de cada caso teniendo en cuenta factores dependientes del paciente (edad, tabaquismo y quizás la ruptura de otro aneurisma), factores del aneurisma (tamaño, ubicación), y el riesgo asumido de la intervención. La decisión debe estar basada en un análisis multidisciplinario del caso individual (clase III, nivel C) . Conclusiones del comentador

Esta patología continúa siendo un tema sometido a continuo debate. Ante un ACNR, de pequeño tamaño, asintomático y ubicado en la circulación anterior se recomienda realizar controles periódicos y controlar los factores de riesgo. Los de mayor tamaño y sobre todos aquellos ubicados en la circulación posterior, serían candidatos a una intervención quirúrgica. Aquellos sintomáticos, independientemente de su tamaño, serían lo más fuertes candidatos a un procedimiento quirúrgico. Estos pacientes deben estar en control y seguimiento estricto por un equipo neuroquirúrgico y su manejo debe ser multidisciplinario.

Mariana Meliné Moreno [ Especialista en Neurología. Sección de Parkinson y Movimientos Anomales del Hospital de Clínicas José de San Martín. yomarianm @ hotmail.com] Moreno M. El curso natural de las aneurismas cerebrales no rotos varía de acuerdo a su tamaño, ubicaci\{on y forma. Evid Act Pract Ambul. 2014:17(3).Jul-Sep. 85. Comentado de: Morita A, Kirino T, Hashi K y col. The Natural Course of Unruptured Cerebral Aneurysms in a Japanese Cohort. NEJM 2012;366:2474-82. PMID: 22738097.

\section{Referencias}

1. Raymond J, y col. Unruptured Intracranial Aneurysms A Critical Review of the International Study of Unruptured Intracranial Aneurysms (ISUIA) and of Appropriate Methods to Address the Clinical Problem Interventional Neuroradiology 2008;14:85-96.

2 Matsumoto K, y col. Investigation of the Surgically Treated and Untreated Unruptured Cerebral Aneurysms of the Anterior Circulation. Surg Neurol 2003;60:516-523. 3 Wiebers DO, y col. Unruptured intracranial aneurysms: natural history, clinical outcome, and risks of surgical and endovascular treatment. International Study of Unruptured Intracranial Aneurysms Investigators. The Lancet 2003;362:103-110.

4 Asari S, Ohmoto T. Natural history and risk factors of unruptured cerebral aneurysms. Clin Neurol Neuro-surg 1993;95:205-14.

5 Juvela S, Porras M, Poussa K. Natural history of un- ruptured intracranial aneurysms: probability of and risk factors for aneurysm rupture. J Neurosurg 2000; 93:379-87. 6 Winn HR, y col. The long-term outcome in patients with multiple aneurysms. J Neurosurg 1983;59:642-51.

7 Yasui N, y col. Subarachnoid hemorrhage caused by previously diagnosed, previously unruptured in- tracranial aneurysms: a retrospective analysis of 25 cases. Neurosurg 1996;39:1096-101. 8 Juvela S y col. European Stroke Organization Guidelines for the Management of Intracranial Aneurysms and Subarachnoid Haemorrhage. Cerebrovasc Disease 2013;35:93-112. 